\title{
Cutting cobalt
}

Renewable energy technologies do not always employ sustainable resources. The scarcity of cobalt supply must be addressed in transportation electrification.

$\mathrm{D}$ riven by unrelenting market demands, especially in electric transportation, rechargeable batteries have become more affordable than ever before. The average price for an electric vehicle (EV) battery pack was over US\$1,100 per kilowatt hour (kWh) in 2010, but it dropped to less than US\$160 per kWh in 2020 (https://about. newenergyfinance.com/electric-vehicleoutlook/). The mileage per charge for EV batteries has similarly increased. Still, EVs in general are more expensive than internal combustion engine vehicles (ICVs). It is commonly understood that the battery cost must fall below US $\$ 100$ per $\mathrm{kWh}$ for future widespread adoption of EVs.

Cobalt is currently the most expensive component used in mainstream passenger EV batteries due to its limited availability. The supply of Co is heavily geographically polarized, with the Democratic Republic of Congo providing over $60 \%$ of the mined cobalt market volume ${ }^{1}$. However, political instability in the country has created a situation in which child labour and environmental damage in Co mining go unimpeded, raising significant ethical concerns about the widespread use of Co in supply chains. How to reduce or even eliminate reliance on Co in EV batteries has long been a hotly pursued topic in both academia and industry.

Over the past decade, two oxide families called layered nickel-manganese-cobalt (NMC) and nickel-cobalt-aluminium (NCA) oxides have dominated the cathode material of choice for passenger EV batteries, primarily because they provide superior energy density over alternatives and thus long drive range. The presence of Co in both the oxides is crucial to maintain their structural stability during battery charge and discharge. Any attempt to reduce or eliminate Co content must therefore address the structural degradation problem while maintaining the capacity of the cathode.

Replacing Co with Ni has long been proposed because not only is $\mathrm{Ni}$ much more abundant, the resultant high-Ni oxides are also expected to yield higher capacities. In practice, however, the electrochemical interplay among these metal cations as well as oxygen anions is so complicated that the cycling stability and thermal stability of high-Ni cathodes have been particularly problematic.
As NMC has a dominant share in the EV industry, tremendous efforts have been made in developing high-Ni NMC by gradually changing the composition of its three elements, from the early NMC111, to NMC532, MNC622 and the latest NMC811 (in this notation, the numbers represent the atomic ratio of Ni:Mn:Co). In 2019, the much-anticipated NMC811 finally made it into commercialization. The NMC811-based battery pack produced by Contemporary Amperex Technology Co. Limited (CATL) delivers an energy density of $170 \mathrm{Wh} \mathrm{kg}^{-1}$, and offers a drive range of over $600 \mathrm{~km}$ for the electric SUV series mass-produced by Nio Inc ${ }^{2}$. Meanwhile, the latest high-Ni NCA battery - mainly made by Panasonic and adapted into Tesla Inc. EVs - uses even less Co than NMC811. The latest Tesla Model 3 equipped with $160 \mathrm{Wh} \mathrm{kg}^{-1} \mathrm{NCA}$ batteries offers a drive range of $560 \mathrm{~km}$ (ref. ${ }^{3}$ ).

Progress in labs continues to be promising. For example, in an Article in this issue of Nature Energy, Yang-Kook Sun, Chong S. Yoon and team develop an oxide cathode $\left(\mathrm{Ni}_{0.9} \mathrm{Co}_{0.09} \mathrm{Ta}_{0.01} \mathrm{O}_{2}\right)$ with even higher $\mathrm{Ni}$ and lower Co content than NMC811, offering a very competitive performance ( $90 \%$ capacity retention in 2,000 cycles and over $850 \mathrm{Wh} \mathrm{kg}^{-1}$ for the cathode).

Complete Co elimination is a more drastic measure to address material availability as it requires very different battery chemistry. Among several Co-free options, recent developments have seen increasing interest in lithium ion phosphate (LFP), originally invented by John Goodenough and colleagues in the 1990s. LFP has an inferior energy density to NMC or NCA, and it was not a strong contender in passenger EV batteries. However, a revival is clearly on the horizon. The first electric motorhome - the Iridium E Mobile made in 2019 - was powered by LFP batteries; 2020 also saw both BYD and Tesla release EVs with the LFP powertrain ${ }^{4}$. More recently, Tesla also announced a new cell design that, by increasing the cell size and removing the tab (that forms the connections between cells), could lead to five times more energy and $16 \%$ greater drive range ${ }^{5}$. This engineering innovation, without changing the LFP chemistry, may make LFP batteries competitive in the market.

Numerous research efforts are also underway to explore new $\mathrm{Co}$-free materials. Much progress on Ni-Mn oxides with various compositions and $\mathrm{Ni}$-rich oxides with various dopants have been reported, although it is too early to predict if these could soon come to the market.

In addition to further battery research and development, recycling spent batteries could contribute to securing Co supply. Despite the current meagre recycling rate for spent $\mathrm{Li}$-ion batteries, there has been growing attention to developing recycling technologies, which will ease the material shortage. It is also worth considering Co sources from non-terrestrial deposits. For example, vast reserves of Co have been reported on the ocean floor and deep-sea mining is on the verge of deployment ${ }^{6}$.

Admittedly, the battery industry won't change overnight. NMC and NCA are likely to maintain their supremacy for the foreseeable future, especially when high energy density is needed. Meanwhile, Co reduction and elimination must remain a pressing need to continue to address its scarcity. With many diverse and exciting developments in tackling the Co issues, we are optimistic about lower-Co and Co-free perspectives. Moreover, the challenge associated with Co reminds us of the need for early consideration of the full product life cycle, including raw material extraction and refinery, fabrication and processing, distribution, operation, and recycling. Ultimately, new energy technology must be designed from the outset for its sustainability.

Published online: 12 November 2020 https://doi.org/10.1038/s41560-020-00731-3

References

1. Lebedeva, N., Di Persio, F. \& Boon-Brett, L. Lithium Ion Battery Value Chain and Related Opportunities for Europe (European Commission, 2016); https://ec.europa.eu/jrc/sites/jrcsh/files/ jrc105010_161214_li-ion_battery_value_chain_jrc105010.pdf

2. Shirouzu, N., Lienert, P. \& Carey, N. The uphill road: battery limitations to test China’s electric vehicle ambitions. Reuters https://www.reuters.com/article/us-autoshow-shanghai-electricinsight/the-uphill-road-battery-limitations-to-test-chinaselectric-vehicle-ambitions-idUSKCN1RS06T (2019).

3. Energy density advances and faster charging would unlock EV revolution. pv magazine https://www.pv-magazine. com/2020/02/11/energy-density-advances-and-faster-chargingwould-unlock-ev-revolution/ (2020).

4. China’s BYD, Tesla release EVs using LFP batteries. Argus https:// www.argusmedia.com/en/news/2108271-chinas-byd-teslarelease-evs-using-lfp-batteries (2020).

5. Lambert, F. Tesla unveils new 4680 battery cell: bigger, $6 \mathrm{x}$ power, and 5x energy. Electrek https://electrek.co/2020/09/22/tesla-4680battery-cell-bigger-power-energy (2020).

6. Shukman, D. Electric car future may depend on deep sea mining $B B C$ News https://www.bbc.co.uk/news/science-environment49759626 (2019). 